\title{
M-Band Ridgelet Transform to Remove Speckle Noise from Medical Images
}

\author{
Ramanjyot Kaur \\ M.Tech Scholar \\ DAVIET, Jalandhar \\ Punjab, India
}

\author{
Palvinder Singh Maan \\ Assistant Professor \\ DAVIET. Jalandhar \\ Punjab, India
}

\begin{abstract}
This paper proposes an improved image denoising algorithm based on M-Band Ridgelet Transform for speckle noise present in the medical images. NeighCoeff Thresholding algorithm is used to calculate the threshold values. The result of the improved method is tested on ultrasound and Magnetic Resonance Imaging (MRI) images affected with speckle noise. Peak Signal to Noise Ratio (PSNR), Mean Square Error (MSE) and Edge Preservation Index (EPI) has been used as parameters for evaluation of results. The performance of new method is compared with existing methods such as Wavelets, Ridgelet, and Curvelet.
\end{abstract}

\section{General Terms}

Image Denoising, Peak Signal to Noise Ratio (PSNR), Mean Square Error (MSE), Edge Preservation Index (EPI)

\section{Keywords}

Speckle noise, Wavelet, Curvelet, Ridgelet.

\section{INTRODUCTION}

With advances in technology, digital images use has been increased in all fields of applications. Considering the real time applications of digital images in medical imaging, two mostly used image forms are ultrasound images and MRI (Magnetic Resonance Imaging) images. The quality of these images depends on their clarity in appearance.

Ultrasound images work on the principle of reflection of sound waves [11]. A fixed frequency of sound waves is passed through patient's body part for which ultrasound scanning has to be performed. From the part being scanned the sound waves are reflected due to difference in the organ's density. The sound waves are reflected as an instance of time. The depth of the organ is measured by the time between the emission of a sound pulse and the reception of its echoes [9]. The final ultrasound image is obtained from the reflected sound waves. Image's poor quality is a major problem in ultrasound images. The main cause of the problem is a kind of noise which is multiplicative in nature and is known as Speckle Noise [15].

\section{SPECKLE NOISE}

Speckle noise occurs due to the reflection of sound waves in the formation of ultrasound images. Speckle noise is multiplicative in nature and usually has a granular appearance. The main cause of speckle noise is the interference between coherent waves which are backscattered by targeted surfaces and arrives out of phase at the receiver side [13].

Speckle noise results in the degradation of ultrasound images by reducing the visual quality and hampering the human observer from seeing the fine details. The detailed visual appearance of ultrasound images is very important in the diagnosis of severe diseases. Therefore, the removal of speckle noise from ultrasound images becomes an important issue. Many filtering techniques have been developed for the removal of speckle noise. Most commonly used filters are Mean and Median Filtering [3], Lee Filter [1], Frost Filter [2] and SRAD Filter [4].

A Mean Filter [3] works by smoothing the data and replacing each pixel with the average of its neighboring pixel values. The process of denoising is equivalent to low pass filtering. The main concept of the mean method is to replace the current pixel value with mean value which is calculated by taking window over the current pixel as the centre [16].

Nonlinear filters are used to remove the speckle noise [12]. The most popular nonlinear filter is the Median Filter [3]. The method used by median filter is to calculate the median of the pixel values taken in a window and the center pixel of the window is replaced with the calculated median value. First, all the pixel values are sorted from the surrounding neighborhood into numerical order and then replacing the pixel being considered with the middle pixel value.

The Lee Filter [1] is another filter which is used to make images free from speckle noise. The lee filter works on the assumption that the mean and variance of the pixel of the interest is equal to the local mean and variance of all pixels within the moving kernel. Lee filter form an output image by computing a linear combination of the center pixel intensity in a filter window with the average intensity of the window. The Lee Filter's design is as such that it eliminates speckle noise while preserving edges and point features in images.

The Frost Filter maintains a balance between Averaging and the All-Pass Filter. The balance is achieved by forming an exponentially shaped filter kernel that can vary from a basic average filter to an identity filter on a point wise, adaptive basis [2]. The results of the filter vary locally with the coefficient of variation.

The Speckle Reducing Anisotropic Diffusion (SRAD)[4] technique is a combination of Lee filter and Frost filters to reduce the speckle noise. SRAD is the edge-sensitive diffusion method for speckled images, in the same way that conventional anisotropic diffusion is the edge-sensitive diffusion for images corrupted with additive noise. The partial differential equations (PDE) approach is used to remove speckle noise [4]. SRAD is efficient in preserving edges and intra region smoothing.

The existing filters for speckle have some limitations. These limitations are:

i) Filters are dependent on the size and shape of the filter's window.

ii) There is smoothing only along the edges and no enhancement of the edges. 
iii) Most of the speckle reducing filters doesn't follow any direction while denoising.

\section{TRANSFORMS USED \\ 3.1 Ridgelet Transform}

\subsubsection{Radon Transform}

The Radon transform [15] of an object $y$ is the collection of line integrals indexed by

$R_{y}(\theta, t)=\int y\left(x_{1}, x_{2}\right) \delta\left(x_{1} \cos \theta+x_{2} \sin \theta-t\right) d x_{1} d x_{2}(1)$

where $\delta$ is the Dirac distribution. The ridgelet coefficients $C R T_{y}(a, b, \theta)$ of an object $y$ are given by analysis of the Radon transform as

$$
C R T_{y}(a, b, \theta)=\int R_{y}(\theta, t) a^{-1 / 2} \psi(t-b / a) d t
$$

\subsubsection{Discrete Ridgelet Transform (DRT)}

A continuous ridgelet transform [13] is calculated by applying one dimensional wavelet transform to the slices of radon transform $\mathrm{R}_{\mathrm{y}}(\theta$,.). Radon transform uses a projection-slice theorem

$\dot{\mathrm{f}}(\omega \cos \theta, \omega \sin \theta)=\int R_{y}(\theta, t) e^{-2 \pi i \omega x} d t$

According to the theorem Radon transform can be obtained by applying the one-dimensional inverse Fourier transform to the two-dimensional Fourier transform of function restricted to radial lines through the origin. For the ridgelet transform, apply a one-dimensional wavelet transform along the radial variable in Radon space. The DRT of an image of size $n \times n$ is an image of size $2 n \times 2 n$, introducing a redundancy factor equal to 4 [5].

\subsection{M-Band Ridgelet Transform (MRT)}

After a signal is decomposed with the 2-band wavelet transform, high frequency channels have wide bandwidth and low frequency channels have narrow bandwidth. These characteristics are not suitable for the signals that have rich middle and high frequency components. The M-band wavelets provide a solution to this problem. The solution to the problem is the combination of the M-band wavelet with the ridgelet transform so that we can have the new transform called M-band ridgelets [12]. M-band ridgelets can be formed by replacing the one Dimensional ordinary wavelet transform with the one Dimensional M-band wavelet transform along the radial variable and then the angular variable. In this way, M-band ridgelet transform possesses the good properties of the ordinary ridgelet transform and the M-band wavelet transform.

Figure 1 shows a flowchart for the digital implementation of proposed M-Band ridgelet transform and the algorithm for the same is given below.

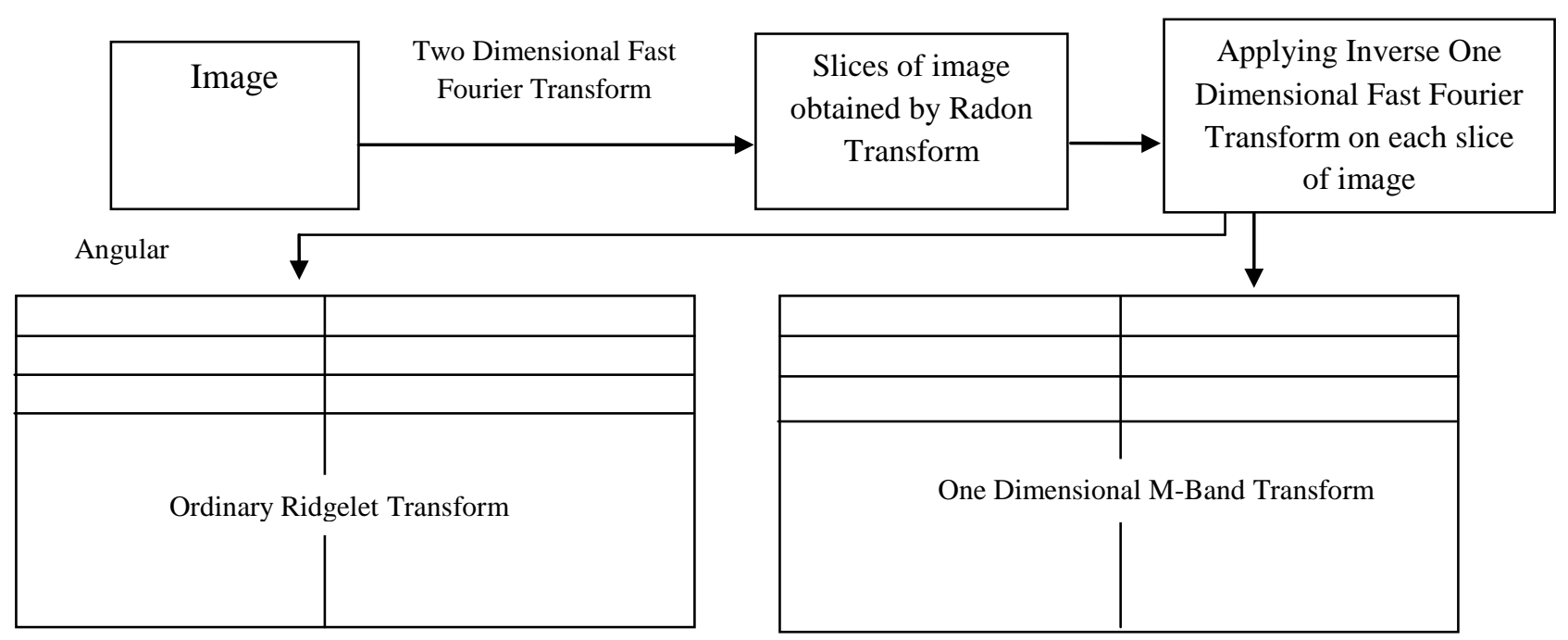

Figure 1: Flowchart of Ridgelet and M-Band Ridgelet Transform

\section{M-BAND RIDGELET BASED} ALGORITHM FOR IMAGE DENOISING

Step 1: Read the original image.

Step 2: Resize the original image to $512 X 512$.

Step 3: Noise is added to the resized image. Noise can be Gaussian noise or Salt and Pepper noise.

Step 4: Apply Â Trous Algorithm [8] to get the Ridgelet coefficients and scaling coefficients.

Step 5: Take one block and apply two dimensional Fast Fourier Transform and perform Radon Transform [7] operation on block.

Step 6: Apply inverse one dimensional fast Fourier Transform on image.
Step 7: Now apply one dimensional Wavelet Transform [10] to obtain the Ridgelet Transform.

Step 8: Choose the threshold by following equations of NeighCoeff Thresholding.

$S^{2_{j . k}}=\sum_{n=-N}^{N} M R T^{2_{j, k+n}} ; N=N_{0}-j$

$M R T_{j, k}=\left\{M R T_{j, k} \mid 1-\frac{\alpha \lambda^{2}}{S^{2} j, k}\right\rfloor$ if $S^{2 j, k} \geq \alpha \lambda^{2}$

Step 9: Now inverse all the process to obtain the denoised image. 


\section{EXPERIMENTAL RESULTS AND DISCUSSIONS}

A number of parameters can be used to evaluate the performance of new and improved image denoising algorithm. In this paper parameters such as Peak Signal to Noise Ratio (PSNR)[6], Mean Square Error (MSE) and Edge Preservation Index (EPI) [9] are used. The comparison of the new improved technique is made with Wavelets [10], Ridgelets [6] [14] and Curvelet [5].

In Figure 2 the sample images taken for experimental purpose are shown

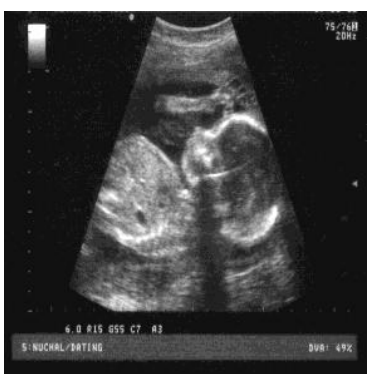

(a)

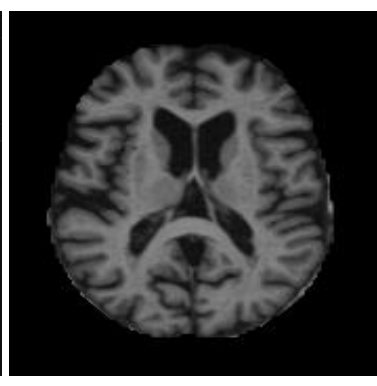

(b)
Figure 2: Sample Images (a) Ultrasound Image (b) MRI image

In Table 1 PSNR values for ultrasound image have been given under Speckle noise with noise density varying from 0.01 to 0.1 .

Table 1: Performance of the proposed algorithm for PSNR values for Ultrasound Image with Speckle Noise

\begin{tabular}{|c|c|c|c|c|}
\hline 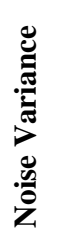 & 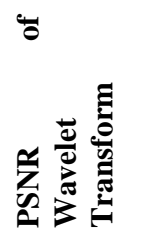 & 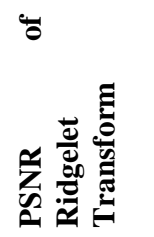 & 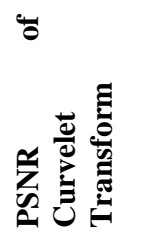 & 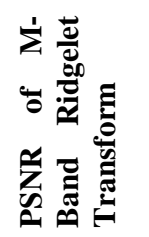 \\
\hline 0.01 & 27.935142 & 25.525584 & 28.747702 & 27.957968 \\
\hline 0.02 & 26.784144 & 24.993698 & 28.544697 & 27.869737 \\
\hline 0.03 & 25.951064 & 24.528920 & 28.360630 & 27.774080 \\
\hline 0.04 & 24.991462 & 24.082212 & 28.188779 & 27.711198 \\
\hline 0.05 & 24.441465 & 23.757703 & 28.009414 & 27.587766 \\
\hline 0.06 & 23.962344 & 23.418891 & 27.861402 & 27.498867 \\
\hline 0.07 & 23.574797 & 23.081802 & 27.730894 & 27.414498 \\
\hline 0.08 & 23.006373 & 22.787907 & 27.534373 & 27.334992 \\
\hline 0.09 & 22.581011 & 22.501140 & 27.388642 & 27.229366 \\
\hline 0.1 & 22.126418 & 22.265937 & 27.233241 & 27.125018 \\
\hline
\end{tabular}

From Table 1, it is seen that the PSNR values of proposed method M-Band Ridgelet Based Transform are better than Wavelet and Ridgelet Transform. The PSNR values of Curvelet and M-Band Ridgelet Transform are nearly equivalent.

In Table 2 MSE values for ultrasound image have been given under Speckle noise with noise density varying from 0.01 to 0.1 .

Table 2: Performance of the proposed algorithm for MSE values for Ultrasound Image with Speckle Noise

\begin{tabular}{|c|c|c|c|c|}
\hline 兽 & 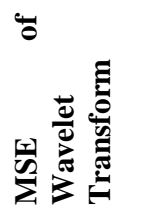 & 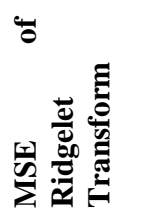 & 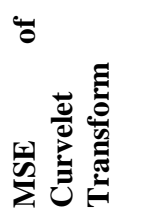 & 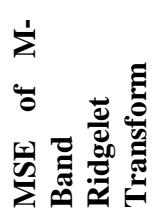 \\
\hline 0.01 & 12.990334 & 13.497738 & 9.314404 & 10.200977 \\
\hline 0.02 & 14.830278 & 14.350276 & 9.534662 & 10.305127 \\
\hline 0.03 & 16.464880 & 15.937844 & 9.738873 & 10.419244 \\
\hline 0.04 & 17.941379 & 16.544553 & 9.933475 & 10.494948 \\
\hline 0.05 & 19.245362 & 17.202660 & 10.140734 & 10.645153 \\
\hline 0.06 & 20.529858 & 17.883400 & 10.315020 & 10.754663 \\
\hline 0.07 & 21.657102 & 18.498855 & 10.471176 & 10.859637 \\
\hline 0.08 & 22.744868 & 19.119793 & 10.710790 & 10.959496 \\
\hline 0.09 & 23.779649 & 19.644607 & 10.892012 & 11.093584 \\
\hline 0.1 & 24.796805 & 24.136864 & 11.088637 & 11.227660 \\
\hline
\end{tabular}

From Table 2 it is seen that the MSE values of improved method are better than Wavelet and Ridgelet Transform.

Table 3: Performance of the proposed algorithm for EPI values for Ultrasound Image with Speckle Noise

\begin{tabular}{|c|c|c|c|c|}
\hline : & 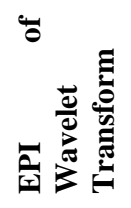 & 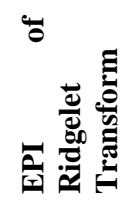 & 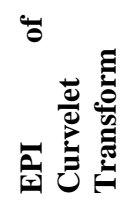 & 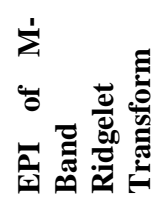 \\
\hline 0.01 & 0.808119 & 0.697284 & 0.669908 & 0.702422 \\
\hline 0.02 & 0.762110 & 0.687972 & 0.670176 & 0.703018 \\
\hline 0.03 & 0.741502 & 0.681091 & 0.667176 & 0.703465 \\
\hline 0.04 & 0.724511 & 0.674344 & 0.665368 & 0.705369 \\
\hline 0.05 & 0.711862 & 0.669681 & 0.662692 & 0.703667 \\
\hline 0.06 & 0.705736 & 0.668446 & 0.658399 & 0.705953 \\
\hline 0.07 & 0.697837 & 0.664920 & 0.658927 & 0.704861 \\
\hline 0.08 & 0.693991 & 0.663541 & 0.654893 & 0.704783 \\
\hline 0.09 & 0.692588 & 0.661615 & 0.655073 & 0.706081 \\
\hline 0.1 & 0.686105 & 0.658383 & 0.652857 & 0.703557 \\
\hline
\end{tabular}

In Table 3 EPI values for ultrasound image have been given under Speckle noise with noise density varying from 0.01 to 0.1 . From Table 3 it is seen that the EPI values of improved method are better than Ridgelet and Curvelet Transform. The M-Band Ridgelet Transform Based Algorithm is efficient in edge preservation.

In Table 4 PSNR values for MRI image have been given under Speckle noise with noise density varying from 0.01 to 0.1 .

Table 4: Performance of the proposed algorithm for PSNR values for MRI Image with Speckle Noise 


\begin{tabular}{|c|c|c|c|c|}
\hline 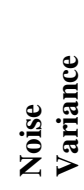 & 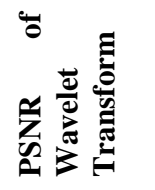 & 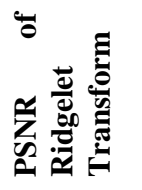 & 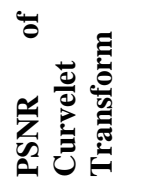 & 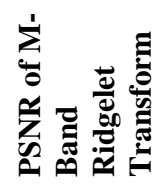 \\
\hline 0.01 & 24.952158 & 1.193642 & 30.471828 & 30.229293 \\
\hline 0.02 & 23.980857 & 7.380640 & 30.096042 & 9254 \\
\hline 0.03 & 23.521583 & 26.384859 & 30.039078 & 29.865730 \\
\hline 0.04 & 23.178900 & 25.541446 & 29.89330 & 29.669898 \\
\hline 0.05 & 22.277705 & 25.389160 & 29.546102 & 2333 \\
\hline 0.06 & 21.973662 & 25.571432 & 29.318011 & 29.453362 \\
\hline 0.07 & 21.898529 & 24.872146 & 29.396860 & 29.200524 \\
\hline 0.08 & 21.201403 & 24.657603 & 29.008312 & 29.219761 \\
\hline 0.09 & 21.221789 & 24.224232 & 28.885504 & 28.977906 \\
\hline 0.1 & 20.874950 & 23.942002 & 28.627657 & 28.784371 \\
\hline
\end{tabular}

From Table 4 it is seen that the PSNR values of M-Band Ridgelet Transform Based Algorithm are better than Wavelet, Curvelet and Ridgelet Transform.

In Table 5 MSE values for MRI image have been given under Speckle noise with noise density varying from 0.01 to 0.1 .

Table 5: Performance of the proposed algorithm for MSE values for MRI Image with Speckle Noise

\begin{tabular}{|c|c|c|c|c|}
\hline 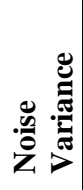 & 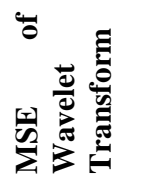 & 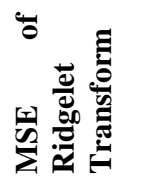 & 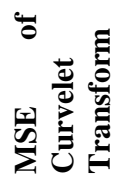 & 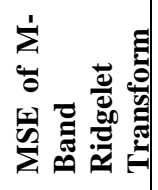 \\
\hline 0.01 & 9.284820 & 6.237482 & 4.432720 & 4.496641 \\
\hline 0.02 & 10.963359 & 7.225280 & 4.691256 & 4.606702 \\
\hline 0.03 & 12.427430 & 8.102961 & 4.911009 & 4.63850 \\
\hline 0.04 & 13.704479 & 8.876402 & 5.122182 & 4.75766 \\
\hline 0.05 & 14.943105 & 9.624874 & 5.364395 & 4.92108 \\
\hline 0.06 & 15.990832 & 10.320114 & 5.575542 & 4.984179 \\
\hline 0.07 & 17.041406 & 10.957058 & 5.762433 & 5.096725 \\
\hline 0.08 & 18.036921 & 11.523544 & 5.990611 & 5.189234 \\
\hline 0.09 & 18.921788 & 12.113081 & 6.147817 & 5.264612 \\
\hline 0.1 & 19.857943 & 12.640171 & 6.333055 & 5.346859 \\
\hline
\end{tabular}

Table 5 shows the MSE values of M-Band Ridgelet Transform Based Algorithm which are better than Wavelet, Ridgelet and Curvelet Transform.

In Table 6 EPI values for MRI image have been given under Speckle noise with noise density varying from 0.01 to 0.1 .

Table 6: Performance of the proposed algorithm for EPI values for MRI Image with Speckle Noise

\begin{tabular}{|c|c|c|c|c|}
\hline Z & 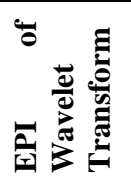 & 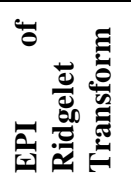 & 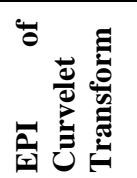 & 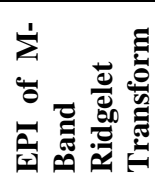 \\
\hline 0.01 & 0.661596 & 0.617682 & 0.582115 & 0.584550 \\
\hline 0.02 & 0.614394 & 0.585496 & 0.563258 & 0.581052 \\
\hline 0.03 & 0.586253 & 0.567607 & 0.551302 & 0.579987 \\
\hline 0.04 & 0.570592 & 0.561788 & 0.546623 & 0.579023 \\
\hline 0.05 & 0.560705 & 0.552562 & 0.537598 & 0.577719 \\
\hline 0.06 & 0.552358 & 0.545801 & 0.535973 & 0.577269 \\
\hline
\end{tabular}

\begin{tabular}{|l|l|l|l|l|}
\hline 0.07 & 0.547557 & 0.542917 & 0.528432 & 0.575201 \\
\hline 0.08 & 0.541652 & 0.539781 & 0.525252 & 0.574596 \\
\hline 0.09 & 0.539158 & 0.534233 & 0.526394 & 0.573061 \\
\hline 0.1 & 0.535841 & 0.536014 & 0.523054 & 0.572030 \\
\hline
\end{tabular}

Table 6 shows the EPI values of M-Band Ridgelet Transform Based Algorithm which are best as compared to the existing methods denoising method, Wavelets, Ridgelets and Curvelet. Thus, it provides better edge preservation.

\section{CONCLUSION}

In this paper M-Band Ridgelet Based Transform Algorithm is proposed for image denoising. The results of the new method have been tested on ultrasound and MRI image. The gathered results are further compared with existing methods such as wavelet, Ridgelet, and Curvelet with parameters PSNR, MSE, and EPI. With analysis of the results it is seen that M-Band Ridgelet Transform provides better results in image denoising. M-Band Ridgelet Transform is best suited to denoise images under speckle noise.

\section{REFERENCES}

[1] Lee, J. S. (1980) "Digital Image Enhancement and Noise Filtering by use of Local Statistics", IEEE Transactions on Pattern Analysis and Machine Intelligence, Vol. PAMI-2, No. 2, pp.165-168.

[2] Frost, V. S., Stiles, J. A., Shanmugan, K. S. and Holtzman, J. C. (1982) "A Model for Radar Images and Its Application to Adaptive Digital Filtering of Multiplicative Noise", IEEE Transactions on Pattern Analysis and Machine Intelligence, Vol. PAMI-4, No. 2, pp. 157-165.

[3] Windyga, P.S. (2001) "Fast Impulsive Noise Removal", IEEE Transactions on Image Processing, Vol. 10. No.1, pp.173-178.

[4] Yu, Y. and Action, S.T. (2002) "Speckle Reducing Anisotropic Diffusion", IEEE Transactions on Image Processing, Vol.11, No.11, pp.1260- 1270.

[5] Starck, J.L. , Candes, E.J. and Donoho, D.L. (2002) "The Curvelet Transform for Image Denoising", IEEE Transactions on Image Processing, Vol.11, No.6, pp.670684.

[6] Do, M.N and Vetterli, M. (2003) "The Finite Ridgelet Transform for Image Representation", IEEE Transactions on Image Processing, Vol. 12, No. 1.

[7] Deans, S. R.(1983) "The Radon Transform and Some of its applications", John Wiley Sons, New York.

[8] Xu, Y.B., Xie, C.S. and Zheng, C.Y. (2007) "An Application of the á Trous Algorithm in Detecting Infrared Targets," IEEE Conference on Wavelet Analysis and Pattern Recognition, Beijing, China, pp. 1015-1019.

[8] Elad, M. and Aharon, M. (2006) "Image Denoising Via Sparse and Redundant Representations Over Learned Dictionaries", IEEE Transactions on Image Processing, Vol. 15, No. 12, pp. 3736-3745.

[9] Joshi, K.R. and Kamathe, R.S. (2008) "SDI: New Metric for Quantification of Speckle Noise in Ultrasound Imaging" IEEE, pp. 122-126.

[10] Hongqiao, L., Shengqian, W. (2009) "A New Image Denoising Method Using Wavelet Transform", IEEE 
International Forum on Information Technology and Applications, pp. 111-114.

[11] Rayudu, D. K. V., Murala, S. and Kumar, V. (2010) "Denoising of Ultrasound Images using Curvelet Transform", The 2nd International Conference on Computer and Automation Engineering (ICCAE 2010), Singapore, Vol. 3, pp. 447-451.

[12] Qiao, Y.L. Song, C.Y. and Zhao, C.H. (2010) "M-band Ridgelet Transform Based Texture Classification," Pattern Recognition Letters, Vol. 31, pp. 244-249.

[13] Gonde, A.B., Maheshwari, R. P. and Balasubramanian, R. (2011) "Multiscale Ridgelet Transform for Content Based Image Retrieval," IEEE $2^{\text {nd }}$ International Advance
Computing Conference (IACC 2010), Thapar University, Patiala, India, pp. 139-144.

[14] Murala, S., Maheshwari, R. P. and Balasubramanian, R. (2011)"Directional Binary Wavelet Patterns for Biomedical Image Indexing and Retrieval", Springer Science.

[15] Li, S., Fang, L. and Yin, H.(2012)“An Efficient Dictionary Learning Algorithm and Its Application to 3-D Medical Image Denoising", IEEE Transactions on Biomedical Engineering, Vol. 59, No. 2, pp. 417-427.

[16] A. Lopes, R. Touzi, and E. Nezry,(1990) "Adaptive speckle filters and scene heterogeneity," IEEE Transactions Geoscience Remote Sensing, Vol. 28, pp. 992-1000. 